Philosophische Fakultät, Universität Ljubljana

Slowenien

urska.valencic-arh@ff.uni-lj.si

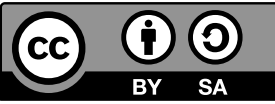

\title{
KINDER- UND JUGENDLITERATUR
}

Gina Weinkauff / Gabriele von Glasenapp (2018): Kinder- und Jugendliteratur. UTB GmbH. Paderborn: Verlag Ferdinand Schöningh. ISBN 978-3-8252-4839-0. Broširano, 320 strani, $22,99 €$.

Mit dem Buch Kinder- und Jugendliteratur legt der Verlag Ferdinand Schöningh einen UTB-Band aus der Reihe Standard Wissen Lehramt - Studienbücher für die Praxis vor. Die Reihe richtet sich an Lehramtstudierenden, Referendare, Lehrerinnen und Lehrer in der Berufseinstiegsphase sowie an alle Fortbildungsteilnehmer in der Ausbildung und in der späteren pädagogischen Tätigkeit. Das Werk der Autorinnen Gina Weinkauff und Gabriele von Glasenapp ist schon auf den ersten Blick ein ansprechendes und besonders für diejenigen, die sich einen elementaren und kompetenten Einblick in das Thema verschaffen wollen, ein anregendes Buch. Der vorliegende Band stellt die aktualisierte und erweiterte 3. Auflage aus dem Jahr 2018 dar. Der ersten Auflage aus 2010 folgte die zweite Auflage in 2014, die in Konzeption und Inhalt nicht verändert wurde, lediglich die Literaturangaben wurden aktualisiert.

Das Werk ist sehr übersichtlich in 10 Kapitel mit mehreren Abschnitten gegliedert. Dem ersten Kapitel vorangestellt ist die Vorbemerkung, in der Autorinnen erklären, diese Einführung in den Teilbereich der Literatur, d.h. in die Kinder- und Jugendliteratur (KJL), „bietet historische und systematische Aspekte“, die auf der Basis des aktuellen Forschungsstandes entstanden sind. Den Begriff der Kinder- und Jugendliteratur stellen sie als ein „soziales Handlungssystem“ vor, das sich vom 18. Jahrhundert an als „ein besonderer Sektor der literarischen Öffentlichkeit“ immer mehr ablöste und verselbständigte. Dieses Werk bietet für die Lehrkräfte nicht nur Grundkenntnisse, sondern ist auch ein guter Begleiter bei didaktischen Überlegungen und ausgezeichneter Wegweiser zur Orientierung im Literaturangebot für Kinder- und Jugendliche.

Kapitel 1 und Kapitel 2 vermitteln Einblicke in zwei Epochen, Aufklärung und Romantik, die KJL nachhaltig geprägt haben. Diese Meinung teilen die Autorinnen mit Seibert (vgl. 2008: 119), der darauf verweist, dass man erst mit der Anerkennung der „Kindheit“ als „eine eigene Phase im Leben eines Menschen“ sowie mit der Realisierung 
des schulischen Unterrichts für alle Kinder vom Beginn der Kinder- und Jugendliteratur sprechen kann. Die Zeit davor wird von der historischen KJL-Forschung erforscht, die u.a. feststellen konnte, dass die Anfänge der KJL bereits ins späte Mittelalter bzw. in die Frühe Neuzeit zurückreichen. Hervorgehoben werden auch Lesegewohnheiten von J.W. v. Goethe, der im Werk Der Orbis sensualium pictus von J. A. Comenius eine sehr anschauliche Freizeitlektüre fand. Für die Entwicklung der eigens für Kinder verfassten Texte in der Aufklärung machte sich die Pädagogik sehr verdient. Die Pädagogen, die den bürgerlichen Habitus verbreiten wollten, erkannten die spezifischen Entwicklungsprozesse der Kinder und die Philanthropen trugen mit dem Prinzip der Kindgemäßheit dazu bei. Im Kapitel 2 steht Romantik als Gegenentwurf zur Aufklärung mit folkloristischen Gattungen und Genres im Vordergrund. Die historischen Aspekte werden durchgehend mit wichtigen Zitaten (die Quellen befinden sich in den Anmerkungen am Ende des Werkes) beweiskräftigt untermauert und vom übrigen Text getrennt. Das Kapitel 3 behandelt realistisches Erzählen aus historischer Sicht und an der Grenze zum phantastischen Erzählen. Realistisches Erzählen definieren die Autorinnen als ,ein mit künstlerischen Mitteln erzielter Effekt" und betonen, dass es dabei nicht „bloß“ um eine Abbildung der Welt oder den Gegenpol zum phantastischen Erzählen geht. Das Kapitel 4 stellt die phantastische Kinderliteratur mit ihren vielen Definitionen vor, deren Ursprünge bereits in der Romantik zu finden sind, sich aber erst in den 1950er und 1960er etablierte. Dieses Genre erfreut sich großer Beliebtheit und wird gut erforscht, wobei die Leistung der Literaturwissenschaftlerin Maria Nikolajevas und der drei zentralen Modelle (die geschlossene sekundäre Welt, die offene sekundäre Welt, die implizierte sekundäre Welt) mit anschließenden Motiven nicht außer Acht gelassen wird. Durch die Impulse aus der Erwachsenenliteratur ist jugendliterarisches Erzählen geprägt, das im Kapitel 5 anhand zweier Modelle mit Subgenres bzw. Ausprägungen behandelt wird: Abenteuerroman und Adoleszenzroman. So wie im gesamten Werk werden auch hier präzise Analysen anhand konkreter Beispiele erarbeitet. Eine der umfangreichsten Analysen und Interpretation vom Gedicht Das ist das Ende von Elisabeth Borchers wird im Kapitel 6 vorgestellt, das sich mit Kinderlyrik und Kindergedicht und vorrangig mit der Notwendigkeit, die beiden Begriffe zu unterscheiden, auseinandersetzt. Das Kapitel 7 steht im Zeichen des Bilderbuches, einer Gattung, die aus verschiedenen Blickwinkeln erfasst und hier in komplexen literarischen, künstlerischen, kulturhistorischen Zusammenhängen verdeutlicht wird. Hervorgehoben wird auch die traditionelle sozialisatorische Funktion, die in der Postmoderne eher in den Hintergrund rückt. Das Bilderbuch kann als Lehrmedium oder als Unterhaltungsmedium betrachtet werden. Die Textstrukturen, die das Bilderbuch bietet, eröffnen Spielräume für verschiedene Lesarten, z.B. Doppelsinnigkeit. Das in der dritten Auflage neu hinzugefügte Kapitel 8 über die Medien der Kinder- und Jugendliteratur befasst sich nicht nur mit medialen, codalen und modalen Besonderheiten der Kinder- und Jugendliteratur, sondern werden in diesem Kapitel auch die historischen Anfänge der Einzelmedien der KJL und der gegenwärtige Medienalltag ausführlich geschildert. Erst seit Mitte der 1970er Jahre kam es zu einer „die Einzelmedien übergreifenden Perspektive“, die als 
Teil der Popkultur im Rahmen der sozialwissenschaftlichen und KJL-Forschungsansätzen entstand. Dabei betonen die Autorinnen, dass sie prinzipiell von der Medialität aller Texte ausgehen. Sie sind an den Medien als Instrumenten zur Verbreitung und Überlieferung von kinder- und jugendliterarischen Texten, die Zeichen aus unterschiedlichen Codes (verbalen, piktoralen, mimischen, gestischen, musikalischen, räumlichen usw.) enthalten können, interessiert. Thematisiert wird die wachsende Präsenz von Computer, Internet und mobilen Geräten, aber nach wie vor besteht eine Vielfalt der Angebote in den traditionellen Einzelmedien. Kapitel 9 beschäftigt sich mit dem Kulturtransfer der KJL im weitesten Sinne, vor allem nach dem Jahr 1945, als man erkannte, dass KJL aus anderen Sprachen und Kulturen zum interkulturellen Verstehen beitragen kann. In den deutschsprachigen Raum werden traditionell viele KJL-Werke aus anderen Sprachen übersetzt, nicht nur die internationalen Klassiker. Es stellte sich heraus, dass der Leser auch sprachliche Veränderungen in der Zielsprache in Kauf nehmen muss. Dieser Tatsache müssen sich die Übersetzer bewusst sein, denn eine Übersetzung ist zunächst eine Interpretation vom Übersetzer, wie Rita Oittinen (2000: 17) feststellt: „The translator is a very special kind of reader: she/he is sharing her/his reading experience with target-language readers." Eine Art Sensibilität für Übersetzungsphänomene im Bereich der KJL entwickelte sich relativ spät, erst nach 1980 und hatte eine kritische Betrachtung zur Folge. Das abschließende Kapitel 10 stellt die KJL im Sozialisationsprozess und in der Schule im historischen Wandel dar. Es wird ausführlich erläutert, wie unterschiedlich die Lektüre von KJL im Laufe der Geschichte betrachtet wurde. Wenn heute literarische Erfahrung und Erwerb vom Weltwissen betont werden, bemängeln die Autorinnen zum Schluss entsprechende didaktische Konzepte zur aktuellen KJL seitens der Literaturdidaktik.

Leserinnen und Leser, die sich einen schnellen Überblick über den Inhalt der einzelnen Kapitel verschaffen wollen, seien auf die letzten Seiten der Kapitel verwiesen. Hier wird das untersuchte Thema, welches zuvor im Detail vorgestellt wird, noch einmal in einem recht prägnant formulierten zusammenfassenden Überblick präsentiert. Jedem Kapitel sind nach der Zusammenfassung mehrere Testfragen angestellt, die der Wissensabfrage dienen und mit denen die LeserInnen die theoretischen Inhalte wiederholen können. Die Lösungen zu den Aufgaben in den jeweiligen Kapiteln, hier als Modellantworten bezeichnet, kann der Leser im Anhang nachschlagen. Zum kreativen Umgang mit verschiedenen Inhalten laden die an die Testfragen anschließenden Anregungen zur Textarbeit ein, die Leser zur wertvollen Weiterarbeit animieren bzw. motivieren. Das Layout ist sehr ansprechend und übersichtlich. Die angeführten Zitate in Kursivschrift heben sich vom übrigen Text ab, was ihre Authentizität und Wichtigkeit zusätzlich unterstreicht.

Das Buch von Gina Weinkauff und Gabriele von Glasenapp bietet insgesamt ein umfangreiches Programm und geht ausführlich auf die wichtigen Fragen im Bereich der KJL ein. Sehr gut ist, dass das Buch nicht nur theoretisch fundiertes Wissen vermittelt, sondern sich um Vermittlung von konkreten Analysen und Interpretationen bemüht sowie zur weiteren Arbeit anregt. Ebenfalls positiv zu bewerten sind die verständliche Sprache, 
die zahlreichen Abbildungen und auch die Anführung von Definitionen aus kanonisierten Werken oder Studien.

Kapitel 1 (Am Anfang war die Pädagogik ...) und Kapitel 2 (Wo Kinder sind, da ist ein goldenes Zeitalter) werden mit kennzeichnenden Sprüchen bzw. verfestigten Zitaten vor dem eigentlichen Titel versehen, die man sich leicht einprägen kann. Schade, dass auch die anderen Kapitel nicht genauso ausgestattet sind. Eine kleine Korrektur würde ich bei der Angabe der Literatur vorschlagen. Es werden Wissenschaftler erwähnt, aber in den Literaturtipps findet man keine weiteren Angaben zu ihren Werken (z.B. Turgay Kulurtay usw. auf S. 205). Etwas irritierend wirkt das kurze Sachregister mit wenigen Begriffen. Wenn das Sachregister die Benutzung des Werkes erleichtern sollte, dann sollte es ausführlicher erfasst werden.

Alles in allem handelt es sich um ein sehr gelungenes Buch, das anschauliches Material zur Kinder- und Jugendliteratur bietet, das in dieser Form bis jetzt auf dem Markt fehlte.

\section{LITERATUR}

OITTINEN, Riitta (2000) Translating for Children. New York, London: Garland Publishing.

SEIBERT, Ernst (2008) Themen, Stoffe und Motive in der Literatur für Kinder und Jugendliche. Wien: Facultas 